\title{
Impacto de la epidemia de neumonía e influenza A H1N1 del 2009 en la tendencia de la mortalidad estandarizada por edad, y en las tasas de muerte específicas por grupos de edad y sexo en México, 2000-2010
}

\author{
David Martínez-Briseño, Ma. Cecilia García-Sancho Figueroa, $₫$ Rosario Fernández-Plata, \\ Luis Torre-Bouscoulet, Laura Suárez González, Rogelio Pérez-Padilla
}

Instituto Nacional de Enfermedades Respiratorias Ismael Cosío Villegas, Ciudad de México.

Trabajo recibido: 20-X-2015; aceptado: 04-XII-2015

\begin{abstract}
RESUMEN. Introducción: El análisis de la mortalidad a nivel nacional permite identificar las principales causas de muerte y los grupos de edad más afectados. Objetivo: Describir el impacto de la epidemia de neumonía e influenza A H1N1 en el año 2009 en la tendencia de la mortalidad estandarizada por edad, y en las tasas de muerte específicas por grupos de edad y sexo en México, 2000-2010. Material y métodos: Se calcularon tasas de mortalidad por neumonía e influenza estandarizadas por edad utilizando el método directo. Los códigos de la Clasificación Internacional de las Enfermedades (CIE) incluidos en esta descripción fueron CIE-10 J09 a J18, se abarcaron todas las edades en el análisis. Para cumplir este objetivo nosotros usamos los datos oficiales de mortalidad (INEGI). Resultados: La mortalidad estandarizada por edad mostró un exceso de muertes debido a neumonías e influenza en el año 2009. Las tasas específicas mostraron que los grupos de edad más afectados fueron en los grupos de edad extremos de la vida. En el 2009, la mortalidad ocurrió también en edades intermedias. Conclusión: El exceso de mortalidad por neumonías e influenza en el 2009 impactó la tendencia de la mortalidad; las tasas de mortalidad por esta causa son altas, sobre todo en menores de 1 año y adultos mayores; se requiere de acciones específicas para disminuirlas.
\end{abstract}

Palabras clave: México, mortalidad, neumonía e influenza A H1N1, tendencias.

ABSTRACT. Introduction: Mortality analysis allows us to identify the main causes of death and the characteristics of affected population. Objective: To estimate the impact of the mortality for pneumonia e influenza A H1N1 in the 2009 on the age-standardised and specific mortality rates in Mexico for 2000-2010. Material and methods: We estimated age-standardised rate by direct method and specific mortality rates due to pneumonia and influenza; the codes CIE-10 J09 aJ18 were analyzed. We used the official database of mortality in Mexico (INEGI). Results: The trend of the age-standardised rates shows an increase in the mortality in 2009, in men and women. The age groups most affected were those over 65 and less than one year. In 2009, the highest rates were observed in middle age group. Conclusion: Deaths due to pneumonia e influenza A H1N1 impacted the age-standardised rates; elderly people and children under one year of age were the most affected. Mortality rates due to pneumonia and influenza are high and particular actions are required to decrease them.

Key words: Mexico, mortality, pneumonia e influenza A H1N1, trends.

\section{INTRODUCCIÓN}

La mortalidad por neumonía e influenza constituye una de las principales causas de muerte en México. De acuerdo con los datos del Instituto Nacional de Estadística y Geografía (INEGI) en el año 2012, las enfermedades respiratorias fueron causas importantes de mortalidad en nuestro país. ${ }^{1}$ Durante el 2009 se documentó una epidemia de neumonía e influenza en México debido al virus de la influenza A H1N1. Aun cuan- do las muertes por neumonía e influenza confirmadas por laboratorio durante el año 2009 fueron escasas, el análisis de la mortalidad nacional por neumonía e influenza durante los últimos 10 años permitió describir el comportamiento epidemiológico de la enfermedad en México. Esta información es básica para la toma de decisiones en salud a futuro.

El objetivo de este estudio fue analizar comparativamente: a) la tendencia de mortalidad anual estandarizada por edad, y b) las tasas anuales específicas por causa 
de muerte, grupo de edad y por sexo durante el período 2000-2010 para las defunciones por neumonía e influenza. La descripción de estos patrones de mortalidad nos permitió identificar las necesidades más apremiantes de prevención y asistencia, así como de entrenamiento médico actuales y futuros en nuestro país.

\section{MATERIAL Y MÉTODOS}

Estudio descriptivo de la mortalidad debido a neumonía e influenza durante el período de 2000 al 2010. La mortalidad fue analizada de dos formas: a) elaborando la tendencia de la mortalidad ajustada (estandarizada) por edad, y b) mediante el análisis de la mortalidad por grupo de edad y sexo. Para cumplir nuestro objetivo se analizaron las bases de datos oficiales de mortalidad para México provenientes del INEGI para el período 2000 a $2010 .^{2}$

Las causas de muerte se agruparon de acuerdo a la Clasificación Internacional de las Enfermedades (CIE10). ${ }^{3}$ Los grupos analizados en este estudio fueron: defunciones por neumonía e influenza (códigos CIE-10 J09 a J18), el análisis incluyó a todos los grupos de edad. Esta agrupación de códigos de la CIE del J09 al J18 para neumonía e influenza fue propuesta por uno de los autores de este trabajo en el 2008. ${ }^{4}$

La tasa estandarizada por edad se calculó por el método directo a partir de los datos oficiales del INEGI, tomando como población estándar la población del año 2010. ${ }^{2}$ Primero fueron calculadas las tasas de mortalidad específicas por año de defunción y edad. A continuación obtuvimos las muertes esperadas multiplicando las tasas de mortalidad específicas a la estructura de población del año 2010. Finalmente, se sumaron las muertes esperadas y se dividieron entre el total de la población de 2010 para los grupos de edad considerados en el estudio. También se calcularon las tasas específicas de muerte anuales para cada grupo por edad y sexo tomando como numerador el número de muertes por año por neumonías e influenza, y como denominador la población de cada año por grupo de edad y sexo para el período 2000-2010. Se usó para el análisis el programa estadístico STATA v. $12 .{ }^{5}$

\section{RESULTADOS}

De 2000 a 2010 se registraron 692,942 defunciones por enfermedades respiratorias del total de 5, 421,059 muertes durante el período (13\%). Del total de muertes por enfermedades respiratorias, el $11.2 \%$ fueron debidas a neumonía e influenza.

El análisis de la mortalidad por neumonía e influenza mostró que la tendencia de la tasa estandarizada por edad permaneció estable de 2000 a 2008, con una tasa de 14 a 15 muertes por 100,000 hombres, con un incremento súbito en la tasa de 17 defunciones por 100,000 hombres durante el 2009, año de la epidemia por influenza A H1N1 en México; en el 2010 las tasas se redujeron nuevamente a 15 muertes por 100,000 hombres. En las mujeres las tasas estandarizadas por edad se mantuvieron con 12 defunciones por 100,000 mujeres, con el incremento en el 2009 a 14 por 100,000 y con un descenso para el año 2010 de 12 defunciones por 100,000 mujeres. Las tasas estandarizadas fueron significativamente más altas en hombres que en mujeres a lo largo de todo el período $(p<0.05)$ (figura 1$)$.

Los resultados del análisis de las causas de muerte específicas por grupo de edad y sexo mostraron que los grupos de edad más afectados fueron los menores de un año y el de 65 y más años, tanto en hombres como en mujeres. Las tasas en hombres fueron más altas que en mujeres; en los hombres, la mortalidad ajustada por edad y sexo mostró unas tasas de 130 a 160 defunciones por 100,000 hombres con 65 y más años y de 120 a 50 defunciones por 100,000 niños menores de un año. En los otros grupos de edad, la mortalidad se mantuvo estable con una tasa de 10 defunciones por 100,000 hombres. A pesar de la reducción importante en la mortalidad infantil ( $<5$ años) que se ha observado en México en las décadas recientes, estas tasas tan altas muestran que la neumonía e influenza continúa siendo una causa importante de muerte en nuestro país. El incremento súbito de la mortalidad en el 2009 se presentó en los hombres de 46-64 años y entre los de 35 a 44 años, es decir, en las edades intermedias de la vida (figura 2).

En las mujeres se observaron tendencias similares, con una tasa de 120 defunciones por 100,000 mujeres

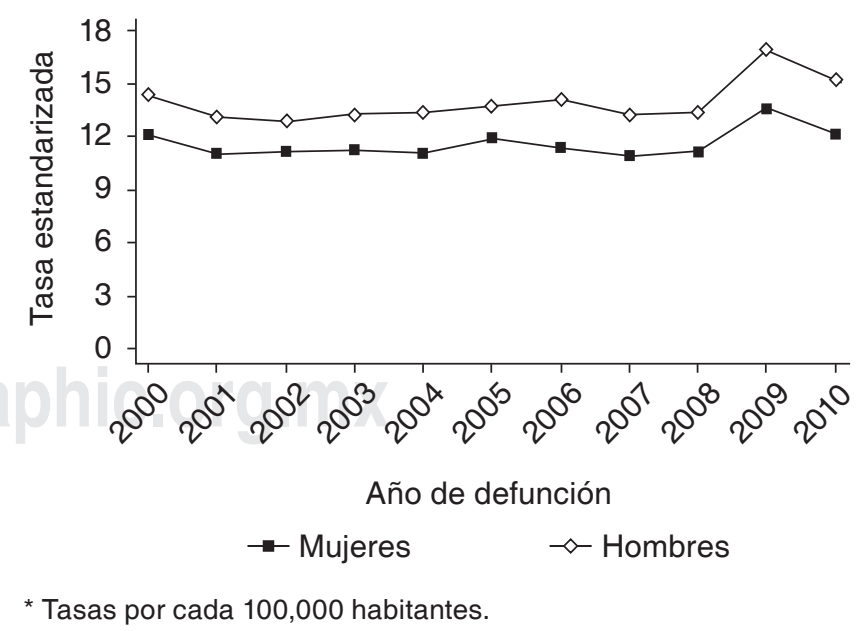

Figura 1. Tasas de mortalidad estandarizadas por edad para la influenza por sexo* 2000-2010. 
en el grupo de 65 y más años y de 90 a 50 muertes por 100,000 menores de un año. El pico de mortalidad en el 2009 se presentó también en las mujeres de 4664 años y entre las de 35 a 44 años, es decir, en las edades intermedias de la vida (figura 2).

\section{DISCUSIÓN}

Los principales resultados de este estudio son: a) la elevada mortalidad debida a neumonía e influenza $A$ H1N1 registrada en el año 2009 impactó la tendencia estandarizada por edad para el período 2000-2010, mostrando un incremento súbito en la mortalidad durante ese año; b) las tasas específicas por edad y sexo mostraron que los grupos más vulnerables son los adultos mayores de 65 y más años y los niños menores de un año; sin embargo, en el año 2009 los grupos más afectados fueron los de edades intermedias y c) la tendencia de la mortalidad estandarizada por edad y el análisis de las tasas anuales específicas por edad y sexo fueron significativamente mayores en los hombres, en comparación con las mujeres.

El virus de la influenza A H1N1 fue responsable de una elevada morbilidad y mortalidad en niños y adultos en todo el mundo. En nuestro estudio observamos que la mortalidad estandarizada por edad debido a neumonías e influenza presentó un incremento súbito durante el año 2009 y un descenso posterior durante el 2010; en ese mismo año, el mayor número de defunciones registradas ocurrieron en edades intermedias de la vida.

Otros investigadores mexicanos han mostrado que concurrentemente a la epidemia por influenza A H1N1, el incremento en la mortalidad de las neumonías durante el 2009 se debió a una epidemia simultánea de neumonía grave. ${ }^{6,7}$ En España hubo también la coexistencia de un aumento en la tasa de neumonía grave con el aumento en las tasas de influenza A H1N1, tanto en zonas urbanas como rurales. ${ }^{8}$ En otro estudio español, las muertes debidas al virus de la influenza A H1N1 ocurrieron sobre todo entre los pacientes con neumonía. En este sentido, el uso temprano de oseltamivir previno las muertes por neumonía. ${ }^{9}$

La tasa de mortalidad por enfermedades respiratorias en varios países de América Latina (Argentina, sureste de Brasil, Chile, Costa Rica, Ecuador, México y Paraguay) había venido descendiendo entre 1998 hasta 2008 en todos los grupos de edad. Esta tendencia a la reducción se revirtió en el año 2009 , quizá como resultado de la pandemia de influenza $\mathrm{A}$ H1N1. ${ }^{10}$

Nuestros resultados también mostraron que los grupos de edad con mayor mortalidad son los menores de un año y los de 65 y más años, tanto para hombres como para mujeres. En el primer grupo de edad se ha reportado de forma asidua la asociación entre la exposición al humo de leña y el incremento en la mortalidad por neumonías en los menores de cinco años a nivel global. ${ }^{11}$ En México, la exposición a humo de leña en

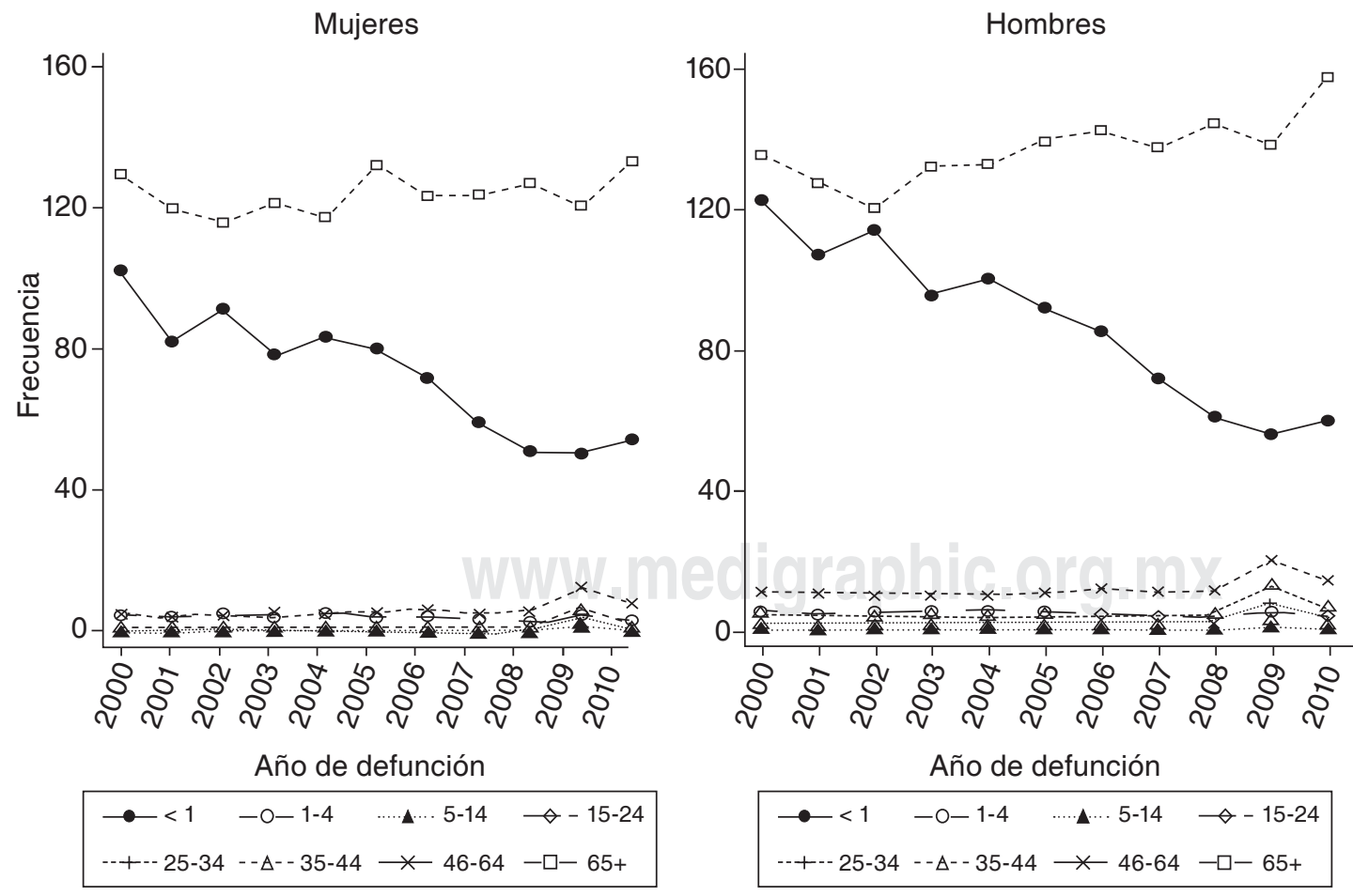

Figura 2.

Muertes anuales por grupo de edad y sexo por influenza. 
el interior de la vivienda sigue siendo elevada ya que, según los datos del INEGI, una entre cinco viviendas en México aún utilizan leña como combustible. ${ }^{12}$

En nuestro estudio la reducción en la tasa de mortalidad por neumonías fue importante durante el período de estudio, de 120 (2000) a 50 defunciones (2010) por 100,000 niños menores de un año. Si bien, y aún cuando nuestros resultados no son comparables con los referidos para el total de América Latina, en esta región se documentó una reducción de las tasas de mortalidad anuales en los menores de cinco años de 56.9 muertes por 100,000 en 1998 a 26.6 muertes por 100,000 niños en 2008. Este estudio también incluyó a México. ${ }^{10}$

La mayor reducción en la tasa de mortalidad en menores de cinco años observada en América latina puede ser debido primero, a los años que se incluyeron en el estudio (1998-2008) y a la diferencia en el grupo de edad estudiado (menores de un año en nuestro estudio en comparación con menores de cinco años en el artículo de América Latina). Sin embargo, las tasas de mortalidad infantil en América Latina y en México siguen siendo elevadas y éstas pueden ser reducidas mediante la eliminación de los factores de riesgo respiratorios (tabaquismo activo, tabaquismo pasivo, exposición a humo de leña, entre otros).

En adultos jóvenes y adultos mayores existen otros factores de riesgo importantes de neumonía e influenza que pueden ser evitados o prevenidos. Para reducir la mortalidad neumonías-influenza, además de la vacunación de grupos vulnerables (niños menores de cinco años, adultos mayores, mujeres embarazadas, adultos jóvenes con factores de riesgo), debe evitarse o reducir las exposiciones a factores de riesgo relevantes, como el tabaquismo y la exposición al humo de leña.

En una cohorte de adultos mayores, el tabaquismo fue un factor de riesgo independiente de mortalidad asociada a influenza. ${ }^{13}$ En México la adicción al tabaquismo es alta. La Encuesta Nacional de Adicciones realizada en el 2011 mostró una prevalencia de consumo activo de tabaco de $21.7 \%$, lo que corresponde a 17.3 millones de mexicanos fumadores. El $31.4 \%$ de los hombres y el $12.6 \%$ de las mujeres son fumadores activos (12 millones de hombres y 5.2 millones de mujeres). Entre el año 2008 y 2011, no se observaron cambios en la prevalencia global o por sexo de consumo activo de tabaco. Al comparar con el año 2002, la prevalencia global de fumadores activos permanece estable $(23.5 \%$ ENA 2002, 21.7\% ENA 2011); aunque, se observa una disminución estadísticamente significativa en el grupo de los hombres (36.2\% ENA 2002, 31.4\%, p < 0.05 ENA 2011). Al analizar el comportamiento de la prevalencia global de fumadores por tamaño de la localidad ésta permanece estable, tanto en el área urbana (26.4\% ENA 2002, 23.3\% ENA 2011) como rural (14.3\% ENA 2002, 16.1\% ENA 2011). ${ }^{14}$

En nuestro estudio, en las dos formas de análisis que utilizamos, los hombres mostraron una tasa mayor de mortalidad en comparación con las mujeres durante todo el período de 2010. La mayor tasa de mortalidad en hombres es consistente con los datos de mortalidad general observados en México por el INEGI. En este estudio, tanto hombres como mujeres presentaron un incremento en la mortalidad en el 2009, la cual se observó en los grupos de edad intermedia. Este hallazgo que ha sido confirmado por otros investigadores implica también la mortalidad materna. En un estudio realizado en México fue constatado que la epidemia de influenza A H1N1 interrumpió la tendencia descendente en la tasa de mortalidad materna que se había venido observando en los últimos años. ${ }^{15} \mathrm{En}$ el 2009 en México se registró la primera muerte materna debido a la epidemia de influenza A H1N1. La defunción se debió a insuficiencia respiratoria aguda. ${ }^{16}$

El análisis de los nuevos factores de riesgo emergentes asociados a mortalidad por neumonías e influenza A H1N1 incluye la obesidad mórbida, el embarazo y las coinfecciones bacterianas. ${ }^{17}$ Otro factor predictor de mortalidad durante la epidemia en México, además de tener una edad intermedia, fue el sobrepeso y obesidad. ${ }^{18}$ Este hallazgo fue consistente con lo observado en Rumania, en donde una cohorte de pacientes con influenza y neumonía mostró que la obesidad fue un factor de riesgo de muerte significativo [RM: 2.9; (IC95\%1.6-31.2)]. ${ }^{19}$

En un estudio realizado en la población derechohabiente del Instituto Mexicano del Seguro Social, el análisis de los datos clínicos de todos los pacientes hospitalizados con el diagnóstico de infección respiratoria aguda mostró que los factores de riesgo de muerte por influenza A H1N1 fueron la diabetes $(R M=1.6)$ y la inmunosupresión $(\mathrm{RM}=2.3)$; mientras que los pacientes asmáticos $(R M=0.3)$ o las mujeres embarazadas $(R M=$ $0.4)$ presentaron menor riesgo de muerte. El efecto del tratamiento antiviral en la mortalidad de los pacientes hospitalizados no alcanzó significancia estadística [RM $=0.56$ (IC95\% 0.29-1.10)] debido, al decir de los autores, a que únicamente el $8.9 \%$ de los pacientes recibieron el tratamiento antiviral. También existió un incremento en el riesgo de muerte en los pacientes hospitalizados con infección A H1N1 con un retraso mayor de dos días desde el inicio de síntomas $(\mathrm{RM}=2.7)$. En el grupo de pacientes con neumonía negativos al virus de la influenza A H1N1 se observaron factores de riesgo de muerte similares. ${ }^{20}$

Nuestro estudio mostró que el análisis de la mortalidad ajustada por edad y las tasas específicas por grupo 
de edad y sexo permitieron describir el comportamiento epidemiológico de la mortalidad por neumonías e influenza en México. Este análisis resultó en una forma útil de identificar problemas de salud prioritarios a partir de las fuentes oficiales de información de mortalidad en México (INEGI).

\section{Limitaciones del estudio}

Una limitación importante de este estudio es que las cifras de mortalidad del INEGI están basadas en las causas registradas en el certificado de defunción; es frecuente que la calidad de la certificación médica de la causa de muerte sea heterogénea dependiendo del personal que certifica la muerte. No obstante lo anterior, la información del INEGI es la mejor fuente de información sobre mortalidad en México.

Una segunda limitación es que nosotros hicimos el análisis tomando en cuenta sólo la causa básica de muerte contenida en el certificado de defunción, lo cual puede subestimar o sobrestimar la mortalidad por algunas causas. Sabemos que el análisis por causa múltiple del certificado de defunción permite conocer de manera más precisa la mortalidad por causa.

\section{CONCLUSIÓN}

La neumonía e influenza es una causa de muerte por enfermedades respiratorias infecciosas muy importante que persiste a pesar de la transición epidemiológica (hacia las enfermedades crónicas) y demográfica (al envejecimiento de la población). Las elevadas tasas de mortalidad en los grupos extremos de la vida, y en nuevos grupos de población vulnerable como los adultos con factores de riesgo requieren de medidas urgentes de intervención. En los próximos años se requerirán recursos adicionales para diagnosticar y tratar las enfermedades respiratorias, así como una mejor preparación del personal de salud.

Ma. Cecilia García-Sancho, David Martínez-Briseño, Rosario Fernández-Plata, y Laura Suárez González participaron en el análisis estadístico del estudio y en la elaboración del manuscrito; Luis Torre-Bouscuolet y José Rogelio Pérez-Padilla participaron en el diseño y análisis del estudio y en la interpretación de los resultados.

\section{REFERENCIAS}

1. Instituto Nacional de Estadística y Geografía. Mortalidad, defunciones generales por grupos de edad $y$ sexo, 2012. Fecha de consulta: 10 de septiembre, 2014. Accesible en: http://www.inegi.org.mx/est/contenidos/ proyectos/registros/ vitales/mortalidad/tabulados/ PC.asp? $\mathrm{t}=14 \& \mathrm{c}=11817$
2. Instituto Nacional de Estadística y Geografía. Mortalidad, defunciones generales por grupos de edad y sexo, 1990 a 2010. Fecha de consulta: 10 de septiembre, 2014. Accesible en: http://www.inegi.org.mx/est/contenidos/ proyectos/registros/vitales/ mortalidad/tabulados/ PC.asp? $\mathrm{t}=14 \& \mathrm{c}=11817$

3. World Health Organization. International Statistical Classification of Diseases and Related Health Problems 10th Revision. Fecha de consulta: September 10, 2014. Available from: http://apps.who.int/classifications/icd10/ browse/2010/

4. Perez-Padilla R. Hidden respiratory disease-associated deaths. Int J Tuberc Lung Dis 2008;12(4):458-464.

5. StataCorp. 2011. Stata Statistical Software: Release 12. College Station, TX: StataCorp LP.

6. Chowell G, Bertozzi SM, Colchero MA, et al. Severe respiratory disease concurrent with the circulation of H1N1 influenza. N Engl J Med 2009;361(7):674-679. doi: 10.1056/NEJMoa0904023.

7. Domínguez-Cherit G, Lapinsky SE, Macias $\mathrm{AE}$, et al. Critically ill patients with 2009 influenza $A(H 1 N 1)$ in Mexico. JAMA 2009;302(17):1880-1887. doi: 10.1001/ jama.2009.1536.

8. Falconer E, Keddy M, Milne WK. Impact of the H1N1 influenza pandemic in two rural emergency departments. Rural Remote Health 2012;12:2063.

9. Viasus D, Paño-Pardo JR, Pachón J, et al.; Novel Influenza $A(\mathrm{H} 1 \mathrm{~N} 1)$ Study Group of the Spanish Network for Research in Infectious Diseases (REIPI). Pneumonia complicating pandemic (H1N1) 2009: risk factors, clinical features, and outcomes. Medicine (Baltimore) 2011;90(5):328-336. doi: 10.1097/ MD.0b013e31822e67a7.

10. de Souza M de F, Widdowson MA, Alencar AP, et al. Trends in mortality from respiratory disease in Latin America since 1998 and the impact of the 2009 influenza pandemic. Bull World Health Organ 2013;91(7):525532. doi: 10.2471/BLT.12.116871.

11. Torres-Duque C, Maldonado D, Perez-Padilla R, Ezzati M, Viegi G; Forum of International Respiratory Studies (FIRS) Task Force on Health Effects of Biomass Exposure. Biomass fuels and respiratory diseases: a review of the evidence. Proc Am Thorac Soc 2008;5(5):577-590. doi: 10.1513/pats.200707-100RP.

12. Instituto Nacional de Estadística y Geografía. Censo de Población y Vivienda 2010. Cuestionario básico. Estados Unidos Mexicanos/Vivienda. Fecha de consulta: 6 de diciembre, 2011. Disponible en: http://www3.inegi.org. $\mathrm{mx} /$ sistemas/Tabulados Básicos).

13. Wong CM, Yang L, Chan KP, et al. Cigarette smoking as a risk factor for influenza-associated mortality: evidence from an elderly cohort. Influenza Other Respir Viruses 2013; 7:531-539. doi: 10.1111/j.17502659.2012.00411.x.

14. Instituto Nacional de Psiquiatría Ramón de la Fuente Muñiz; Instituto Nacional de Salud Pública; Secretaría de Salud. Encuesta Nacional de Adicciones 2011: Reporte de Tabaco. Reynales-Shigematsu LM, Guerrero-López CM, Lazcano-Ponce E, et al. México DF, México: INPRFM, 
2012. Disponible en: www.inprf.gob.mx, www.conadic. gob.mx, www.cenadic.salud.gob.mx, www.insp.mx).

15. Fajardo-Dolci G, Meljem-Moctezuma J, VicenteGonzález E, et al. Analysis of maternal deaths in Mexico occurred during 2009. Rev Med Inst Mex Seguro Soc 2013;51(5):486-495.

16. Noguera Sánchez MF, Karchmer Krivitzky S, EsliRabadán MC, Antonio Sánchez P. Case report of the first world death due to a new strain of human influenza A H1N1 virus and behavior of human influenzae in pregnant women. Ginecol Obstet Mex 2013;81(1):47-51.

17. Punpanich W, Chotpitayasunondh $\mathrm{T}$. A review on the clinical spectrum and natural history of human influenza. Int J Infect Dis 2012;16(10):e714-e723. doi: 10.1016/j. ijid.2012.05.1025.

18. Cruz-Licea V, González-Domínguez F, Avila G, Flisser A. Flu symptoms and preventive measures practiced by the inhabitants of Mexico City during the AH1N1 influenza epidemic. Rev Invest Clin 2013;65(4):284-290.

19. Zolotusca L, Jorgensen $P$, Popovici O, et al. Risk factors associated with fatal influenza, Romania, October 2009. May 2011. Influenza Other Respir Viruses 2014;8(1):8-12. doi: 10.1111/irv.12209.
20. Chowell G, Echevarría-Zuno S, Viboud C, et al. Epidemiological characteristics and underlying risk factors for mortality during the autumn 2009 pandemic wave in Mexico. PLoS One 2012;7(7):e41069. doi: 10.1371/ journal.pone.0041069.

\section{$\triangle$ Correspondencia:}

Dra. en C. Ma. Cecilia García-Sancho Figueroa, Instituto Nacional de Enfermedades Respiratorias Ismael Cosío Villegas. Calzada de Tlalpan Núm. 4502, Col. Sección XVI, 14080, Ciudad de México. Teléfono: 54-87-17-00, extensión 5238; fax: $56-65-46-23$ Correo electrónico: cegarsan@netscape.net; cegarsanfi@gmail.com Los autores declaran no tener conflicto de intereses. 